\title{
Moving away from singly-magic nuclei with Gorkov Green's function theory
}

\author{
V. Somà ${ }^{1}$, C. Barbieri ${ }^{2}$, T. Duguet ${ }^{1,3}$, and P. Navrátil ${ }^{4}$ \\ 1 IRFU, CEA, Université Paris-Saclay, 91191 Gif-sur-Yvette, France \\ 2 Department of Physics, University of Surrey, Guildford GU2 7XH, United Kingdom \\ 3 KU Leuven, Institut voor Kern- en Stralingsfysica, 3001 Leuven, Belgium \\ 4 TRIUMF, 4004 Westbrook Mall, Vancouver, BC, V6T 2A3, Canada
}

\begin{abstract}
Ab initio calculations of bulk nuclear properties (ground-state energies, root mean square charge radii and charge density distributions) are presented for seven complete isotopic chains around calcium, from argon to chromium. Calculations are performed within the Gorkov self-consistent Green's function approach at second order and make use of two state-of-the-art two- plus three-nucleon Hamiltonians, $N N+3 N(\operatorname{lnl})$ and $\mathrm{NNLO}_{\text {sat }}$. An overall good agreement with available experimental data is found, in particular for differential energies (charge radii) when the former (latter) interaction is employed. Remarkably, neutron magic numbers $N=28,32,34$ emerge and evolve following experimental trends. In contrast, pairing gaps are systematically underestimated. General features of the isotopic dependence of charge radii are also reproduced, as well as charge density distributions. A deterioration of the theoretical description is observed for certain nuclei and ascribed to the inefficient account of (static) quadrupole correlation in the present many-body truncation scheme. In order to resolve these limitations, we advocate the extension of the formalism towards incorporating breaking of rotational symmetry or, alternatively, performing a stochastic sampling of the self-energy.
\end{abstract}

\section{Introduction}

A leap forward in ab initio calculations of atomic nuclei occurred about 15 years ago with the (re)introduction, in nuclear structure theory, of so-called correlation expansion methods [1, 2]. As opposed to virtually exact approaches, which do not impose any formal approximation on the solution of the many-body Schrödinger equation and scale exponentially or factorially with the system size, correlation expansion techniques achieve a polynomial scaling at the price of an approximate, yet controlled and systematically improvable, solution. Combined with the availability of "softer" Hamiltonians, obtained via similarity renormalisation group (SRG) transformations [3], such a favourable scaling progressively enabled the extension of first-principle calculations beyond the region of light nuclei traditionally targeted by ab initio practitioners. Nowadays, systems up to mass number $A \sim 70$ can be routinely accessed $[4,5,6,7,8]$, with a few attempts reaching out to neutron-deficient tin $(A \sim 100)[9,10]$ or even neutron-rich tin and xenon $(A \sim 140)$ [11] nuclei.

Many-body theories accessing mid-mass nuclei standardly expand the exact ground-state wave function with respect to a reference Slater determinant and can thus efficiently access doubly closed-shell systems dominated by dynamical, i.e. weak, correlations. However, the restriction to a single symmetry-restricted reference product state is too limiting to generate a meaningful expansion in open-shell nuclei due to the associated degeneracy with respect to elementary particle-hole excitations. The use of more general reference states must be contemplated to lift the degeneracy and tackle, from the outset, strong static correlations characterising open-shell systems. To this purpose, three different strategies have been explored so far. The first option relies on reference states mixing a set of appropriately chosen Slater determinants [12, 13]. Those multi-configurational reference states can, for example, be obtained from a prior no-core shell model calculation [14] in a small basis or under the form of a particlenumber-projected Hartree-Fock-Bogolyubov (HFB) state. Such reference states have been successfully employed in the multi-reference extension of the in-medium similarity renormalisation group method $[5,15,16]$ or within a perturbative framework yielding multi-configurational perturbation theory [17]. The second possibility consists in using a doubly closed-shell nucleus as a core, and computing the valence-space interaction at play on top of it through a polynomially-scaling method. Subsequently, a factorially-scaling diagonalisation is performed to solve the Schrödinger equation within the valence space to a high degree of precision $[18,19,20]$. While this method benefits from the maturity of the shell-model technology, its hybrid numerical scaling limits its applicability to nuclei traditionally accessible by the shell model, i.e. $A \lesssim 100$. 
A third route, followed here, relies on the use of a reference product state breaking one or several symmetries of the underlying Hamiltonian. In doing so, one can trade the degeneracy with respect to particle-hole excitations characterising open-shell systems for a degeneracy with respect to transformations of the associated symmetry group. As a result, the particle-hole degeneracy is lifted and a well-defined many-body expansion on top of a "deformed" reference product state can be designed. This trade-off allows one to access open-shell systems while maintaining a polynomial cost and the intrinsic simplicity of singlereference expansion methods. The handling of the pseudo Goldstone mode associated with the manifold of degenerate states, necessary to restore the broken symmetry, can be safely postponed to a later stage [21, 22, 23].

Largely employed in the context of nuclear energy density functional [24], this approach was imported in ab initio nuclear structure about a decade ago. First, Gorkov self-consistent Green's function (GSCGF) theory was developed [25]. Few years later, coupled cluster theory was extended to the use of a Bogolyubov reference state [26]. More recently, Bogolyubov many-body perturbation theory (BMBPT) was introduced as a generalisation of standard Møller-Plesset theory [27, 28]. All these techniques rely solely on the breaking of the U(1) symmetry related to particle-number conservation and are thus designed to efficiently account for static pairing correlations. In order to deal with the other source of strong correlations in nuclei, i.e. the quadrupole correlations typically associated with nuclear deformation, one would need to correspondingly break rotational $\mathrm{SU}(2)$ symmetry. Although work in this direction is in progress (see e.g. [29, 30]), the latter feature is currently unavailable in nearly all state-of-the-art implementations. As a consequence, the above methods are preferentially applied to singly open-shell (i.e., semimagic) nuclei, where the role of quadrupole correlations is not predominant. Indeed, GSCGF and BMBPT have successfully addressed complete semi-magic isotopic chains, e.g. oxygen, calcium or nickel [31, 32, 33, 27, 8, 28]. The limits of applicability of U(1)-breaking, $\mathrm{SU}(2)$-conserving correlation expansion methods, however, have never been systematically probed. Therefore, it is worthwhile to push such calculations away from semi-magic nuclei in order to empirically identify if and where such a strategy eventually fails, i.e. the point beyond which an explicit breaking of $\mathrm{SU}(2)$ symmetry will become mandatory.

Recently, specific medium-mass doubly open-shell systems, e.g. some titanium [34] or sulfur and argon [35, 36] isotopes, have been computed within the GSCGF approach. In this paper, we extend these works to a systematic study of several isotopic chains around semi-magic calcium for which results were not available before. In particular, we compute ground-state energies, charge radii and selected charge density distributions for chains ranging from argon $(Z=18)$ to chromium $(Z=24)$ and compare to available experimental data. Calculations were performed using the recently introduced $N N+3 N(\operatorname{lnl})$ Hamiltonian [8]. For charge radii and densities, additionally, the $\mathrm{NNLO}_{\text {sat }}[37]$
Hamiltonian was employed. Overall, the goals of the present study can be summarised as follows:

1. Assess the performance of state-of-the-art ab initio calculations on bulk properties of medium-mass nuclei. In this respect, the present work follows up on the results of Ref. [8], in which the novel $N N+3 N(\operatorname{lnl})$ interaction was benchmarked on semi-magic oxygen, calcium and nickel isotopes. Here it is shown that the global satisfactory agreement with experimental data found in Ref. [8] extends to doubly open-shell isotopes around calcium. Remarkably, neutron magic numbers $N=28,32,34$ emerge and evolve following experimental trends. While the neutron dripline is not addressed here, the proton dripline is found at or near the experimental one. As already remarked in Ref. [8] for calcium, charge radii computed with $N N+3 N(\operatorname{lnl})$ are too small compared to the experimental values. In contrast, $\mathrm{NNLO}_{\text {sat }}$ provides a good overall description of existing data. Nevertheless, even our best calculations fail to reproduce some finer details, e.g. the steep rise between $N=28$ and $N=32$ and the parabolic-like behaviour between $N=20$ and $N=28$. The latter can be in part ascribed to many-body truncations. Interestingly, for both interactions, a second, smaller, kink is observed at $N=34$.

\section{Analyse pairing properties in nuclei within a first-} principle description.

The ability of accessing ground-state energies of oddeven nuclei enables the investigation of pairing effects e.g. by considering three-point mass differences in even$Z$ isotopic chains. The resulting pairing strength turns out to be underestimated compared to experimental observations, which possibly points to missing manybody correlations.

3. Probe the limits of SU(2)-conserving correlation expansion methods in the description of doubly open-shell nuclei.

It is observed that the description of experimental data deteriorates for certain sets of nuclei away from singlymagic calcium. It is conjectured that this might signal the onset of significant quadrupole correlations, i.e. static deformation. A careful scrutiny indeed reveals a correlation between the inaccuracy of the results (quantified in terms of deviation from experimental data) and an estimate of the deformation.

Developing the above points, the manuscript is organised as follows. First, the theoretical and computational scheme is briefly recalled in Sec. 2. Section 3 is devoted to the study of ground-state energies, in the form of either total (Sec. 3.1) or differential (Secs. 3.2 and 3.3) binding energies. Further, a discussion of three-point mass differences is presented in Sec. 3.4. The impact of (expected) nuclear deformation on calculated ground-state energies is investigated in Sec. 3.5. Finally, a systematic survey of nuclear radii and a selection of representative charge density distributions are presented in Sec. 4. Conclusions and perspectives follow in Sec. 5. 


\section{Computational set-up}

All calculations presented here were performed within the Gorkov self-consistent Green's function approach at second order in the algebraic diagrammatic construction expansion $[\mathrm{ADC}(2)][25,38]$. An extensive study of oxygen, calcium and nickel isotopes has been recently carried out in the same computational scheme and published in Ref. [8]. Hence, only the most salient features are recalled here and the reader is referred to [8] for more computational and technical details.

Two different two- plus three-nucleon $(2 \mathrm{~N}+3 \mathrm{~N})$ Hamiltonians were employed in the present study. The first one, labelled $N N+3 N(\operatorname{lnl})$, is based on the next-to-next-tonext-to-leading order $\left(\mathrm{N}^{3} \mathrm{LO}\right)$ nucleon-nucleon potential from Entem and Machleidt [39, 40] complemented with the $\mathrm{N}^{2} \mathrm{LO} 3 \mathrm{~N}$ interaction for which a combination of local and nonlocal regulators is used [8]. Low-energy constants were fitted to $A=2,3,4$ systems. This Hamiltonian is further SRG-evolved to a low-momentum scale of $\lambda=2 \mathrm{fm}^{-1}$. The second one, labelled $\mathrm{NNLO}_{\text {sat }}$, was introduced in Ref. [37] with the explicit goal of providing an improved description of saturation properties. Here, in contrast to $N N+3 N(\operatorname{lnl})$, low-energy constants were simultaneously fitted to fewbody systems as well as selected ground-state energies and radii of carbon and oxygen isotopes. This Hamiltonian is SRG-unevolved.

Three-nucleon forces are treated following the formalism developed in Ref. [41]. In practice, the three-body Hamilton operator is self-consistently convoluted with the correlated one-body density matrix and contributes to one- and two-body effective interactions [42]. The contributions resulting from contracting two- and many-body density matrices were seen to be negligible for our purposes $[43,44]$. Note that we discard interaction-irreducible diagrams containing three-body vertices. The formalism needed to include these at the $\operatorname{ADC}(3)$ level was presented in Ref. [45] and their contribution is estimated to be comparable, in terms of both importance and required computing resources, to $\mathrm{ADC}(5)$ computations with only two-nucleon interactions. The procedure used in this paper generates an $A$-dependent symmetry-conserving Hamiltonian that can be viewed as a generalisation of the particle-numberconserving normal-ordered two-body approximation discussed in Ref. [46].

As for (many-body) operators, they are expanded on a one-body harmonic oscillator (HO) basis (or products thereof) including states up to $e_{\max } \equiv \max (2 n+l)=$ 13. Three-body operators are further restricted to threebody basis states characterised by $e_{3 \max }=16<3 e_{\max }$. For some representative isotopes, a variation of the $\mathrm{HO}$ frequency $\hbar \omega$ was performed in order to locate the optimal value for the binding energy and radius, which are observables considered in this work. Based on this analysis, we performed calculations using $\hbar \omega=18 \mathrm{MeV}$ for both energies and radii for $N N+3 N(\operatorname{lnl})$, and $\hbar \omega=20 \mathrm{MeV}$ and $\hbar \omega=14 \mathrm{MeV}$ for energies and radii respectively for $\mathrm{NNLO}_{\text {sat }}$. All results presented here were obtained with these model space parameters, unless otherwise stated.
In Ref. [8] theoretical uncertainties associated to modelspace and many-body truncations were investigated for calcium isotopes. For total ground-state energies, such uncertainties were estimated to be respectively $0.5 \%$ and $2 \%$ $(1 \%$ and $3 \%)$ for $N N+3 N(\operatorname{lnl})\left(\mathrm{NNLO}_{\text {sat }}\right)$. For charge radii, a combined (model space plus many-body) uncertainty of $0.1 \mathrm{fm}$ was assumed for both interactions. A refined uncertainty analysis for charge radii is presented in Sec. 4. Even if the estimates on many-body truncation will have to be corroborated by explicit GSCGF-ADC(3) calculations in the future, one can suppose that the above values give a reasonable indication also for the (doubly) open-shell nuclei considered here.

\section{Ground-state energies}

\subsection{Total energies}

Let us start by analysing total ground-state energies along the seven isotopic chains studied in this work, i.e. argon $(Z=18)$, potassium $(Z=19)$, calcium $(Z=20)$, scandium $(Z=21)$, titanium $(Z=22)$, vanadium $(Z=23)$ and chromium $(Z=24)$. The current implementation of GSCGF theory is based on the assumption that $J^{\Pi}=0^{+}$ for targeted ground states and is therefore well suited for

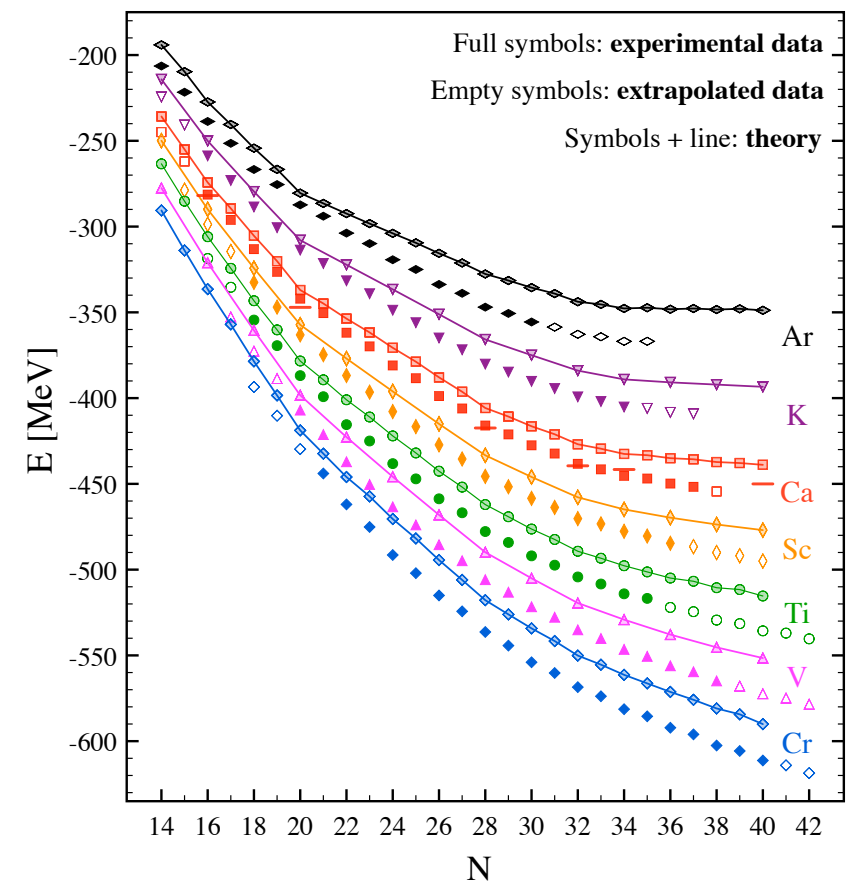

Fig. 1. Total binding energies along $Z=18-24$ isotopic chains computed at the $\mathrm{ADC}(2)$ level with the $N N+3 N(\operatorname{lnl})$ interaction (symbols joined by solid lines). For comparison, experimental data (measured [47, 34, 48, 49, 36], full symbols and extrapolated [47], empty symbols) are displayed. Both calculated and experimental values are shifted by $(20-Z) \times 20$ $\mathrm{MeV}$ for a better readability. For closed-shell calcium isotopes, available $\mathrm{ADC}(3)$ results [8] are displayed as horizontal lines. 
even-even nuclei. The ground-state energy of odd-even systems can be computed via [50]

$$
E_{\text {odd-even }}^{A}=\tilde{E}^{A}+\omega_{0},
$$

where $\tilde{E}^{A}$ is the ground-state energy of the odd-even nucleus computed as if it had $J^{\Pi}=0^{+}$, i.e. as a fully paired even-number-parity state forced to have the right odd number of particles on average, and $\omega_{0}$ is the lowest one-nucleon separation energies in the latter calculation. Further details can be found in Refs. [25, 38]. A more direct but similar approach is to use the addition and separation energies encoded in the spectral function but to recompute the even-even isotope with the center of mass corrections for $A \pm 1$, as done in Ref. [42]. As a result, one can access the ground-state energy of all isotopes with even $Z$ and that of odd-even isotopes with odd $Z$. Other observables, e.g. radii or densities, are instead available only for even-even systems. Further developments, e.g. involving the use of Hellmann-Feynman theorem, are needed to extend their calculation to odd-even systems.

Computed ground-state energies are presented in Fig. 1 and compared to experimental (measured and extrapolated) data. The global behaviour is well captured by the calculated energies across all values of $Z$ and $N$, although underbinding with respect to experiment is observed for all chains. The deviation per nucleon is roughly of the same magnitude for all nuclei (as also visible from Fig. 8, discussed below). This points to a global effect like e.g. missing specific many-body correlations. Indeed, $\mathrm{ADC}(3)$ results for calcium isotopes [8], displayed in Fig. 1 as horizontal bars, show that excellent agreement with experimental values is reached once a more refined truncation schemes is used. These findings confirm the good performance achieved by the $N N+3 N$ (lnl) Hamiltonian for ground-state energies in this mass region [8].

\subsection{One- and two-nucleon separation energies}

Systematically accessing successive nuclides along isotopic or isotonic chains allows to investigate some of the most fundamental properties of atomic nuclei such as the limits of their existence as bound states or the emergence (and evolution) of magic numbers. Such properties are best studied by looking at total ground-state energy differences. Two-neutron separation energies

$$
S_{2 \mathrm{n}}(N, Z) \equiv|E(N, Z)|-|E(N-2, Z)|
$$

are first considered. Their values computed from the total energies of Fig. 1 are shown in Fig. 2, together with available and extrapolated experimental data. The overall agreement with experiment is remarkable, with computed values following the main trends of measured data. The two neutron magic numbers $N=20$ and $N=28$, associated with sudden drops of $S_{2 \mathrm{n}}$, are visible in all theoretical curves. The $N=28$ gap is very well reproduced across all isotopic chains, with the good description carrying over to larger neutron numbers for most chains. On the contrary,

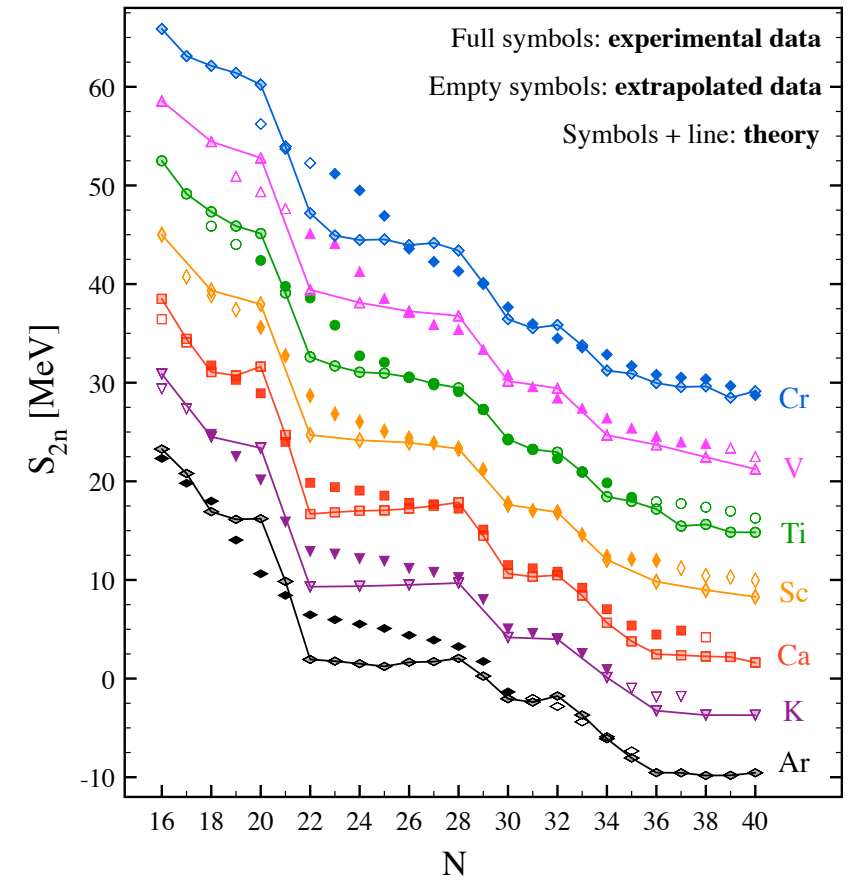

Fig. 2. Two-neutron separation energies along $Z=18-24$ isotopic chains computed with the $N N+3 N(\operatorname{lnl})$ interaction (symbols joined by solid lines), compared to experimental (measured, full symbols and extrapolated, empty symbols) data. Both calculated and experimental values are shifted by $(Z-20) \times 5$ $\mathrm{MeV}$ for a better readability.

the gap at $N=20$ turns out to be overestimated, with the comparison to experiment worsening when departing from proton magic number $Z=20$. The description deteriorates also in other regions, e.g. for argon isotopes between $N=20$ and $N=28$ or more generally for chromium isotopes. In such systems both protons and neutrons have an open-shell character. The absence of a closed shell is likely to induce strong quadrupole correlations that are difficult to capture in the present calculations, based on expanding over a spherical reference state.

The neutron dripline, i.e. the position of the last bound system in a given isotopic chain, can be also read from two-neutron separation energies as unbound nuclei are characterised by negative values of $S_{2 n}$. None of the computed neutron rich isotopes shown in Fig. 2 results unbound, i.e. the dripline is predicted to be located beyond $N=40$ for all considered chains ${ }^{1}$. The smallest $S_{2 n}$ value are reached for ${ }^{56-57} \mathrm{Ar}$ and are as low as $100 \mathrm{keV}$. However, one must remark that continuum coupling is likely to play an important role when binding energies are so close to the neutron emission threshold. Presently, the continuum is crudely included via the discretised harmonic oscillator basis, which does not ensure correct asymptotic properties. In future studies, in order to reliably determine the position of the

1 Present calculations could not be extended beyond $N=40$ due to convergence issues, see discussion in Ref. [8] for more details. 
neutron dripline, particular care will have to be devoted to a more proper treatment of this aspect.

The coupling to the particle continuum plays a lesser role around the proton dripline because of Coulomb repulsion. Given that present calculations span several neighbouring chains, the proton dripline can be investigated within this theoretical setting. Here, the key quantities are one-proton and two-proton separation energies, defined respectively as

$$
S_{1 \mathrm{p}}(N, Z) \equiv|E(N, Z)|-|E(N, Z-1)|
$$

and

$$
S_{2 \mathrm{p}}(N, Z) \equiv|E(N, Z)|-|E(N, Z-2)| .
$$

For a given element, the most proton-rich isotope for which both $S_{1 \mathrm{p}}>0$ and $S_{2 \mathrm{p}}>0$ determines the position of the proton dripline. In Fig. 3, measured and computed $S_{1 \mathrm{p}}$ and $S_{2 \mathrm{p}}$ are displayed as a function of neutron number for the isotopic chains considered in this study ${ }^{2}$. Experi-

\footnotetext{
${ }^{2}$ For potassium only $S_{1 \mathrm{p}}$ can be computed, while for argon none of the two separation energies is available in the present calculations.
}

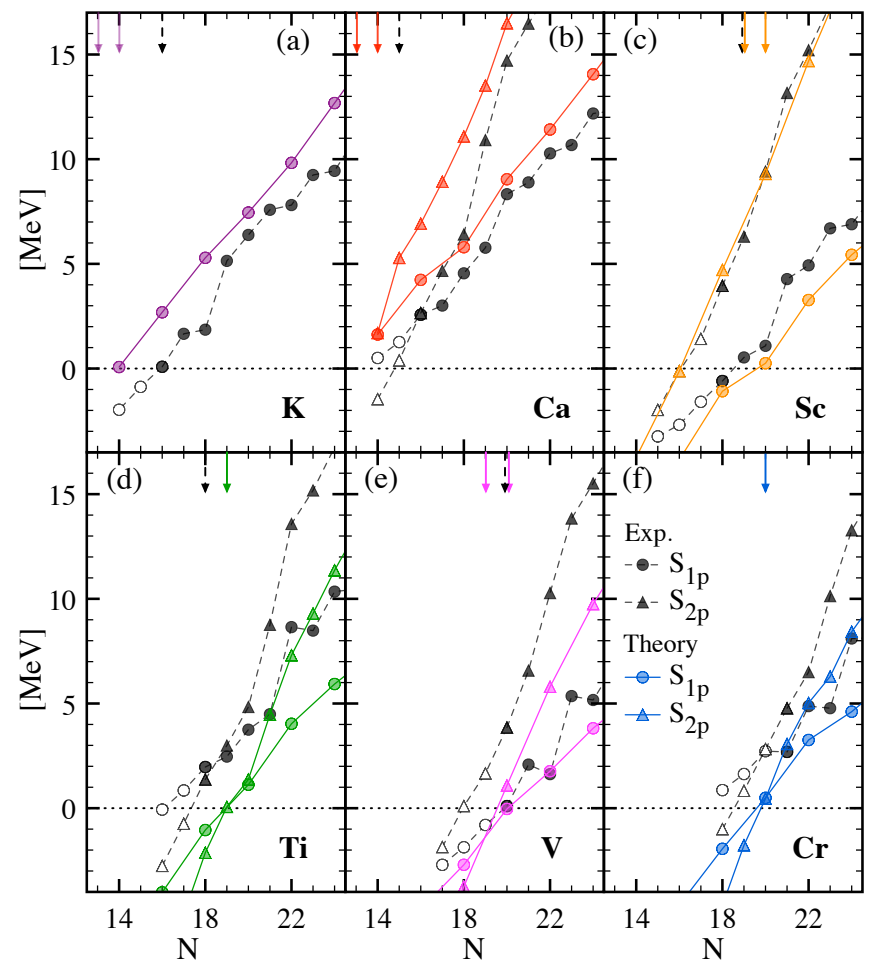

Fig. 3. One- and two-proton separation energies displayed as a function of neutron number for different $Z$. Calculations performed with the $N N+3 N(\operatorname{lnl})$ interaction (symbols joined by solid lines) are compared to existing data (measured, full symbols and extrapolated, empty symbols, all joined by dashed lines). The solid coloured (dashed black) arrows at the top of each panel mark the computed (experimental) driplines. In some cases $(\mathrm{K}, \mathrm{Ca}, \mathrm{Sc}, \mathrm{V})$ the theoretical dripline can not be determined unambiguously from the calculations, hence the two possible values are shown.

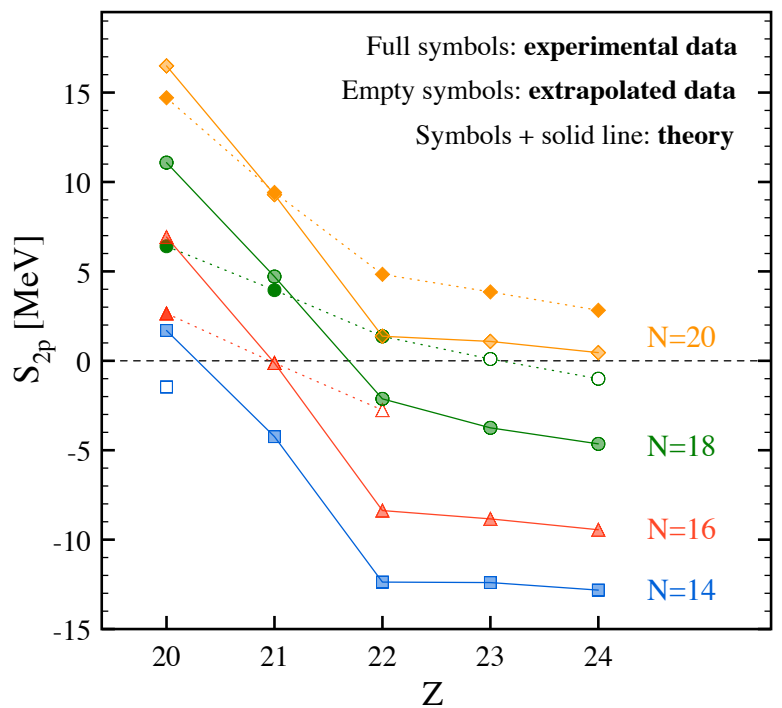

Fig. 4. Two-proton separation energies displayed as a function of proton number for different isotonic chains. Calculations performed with the $N N+3 N(\operatorname{lnl})$ interaction (symbols joined by solid lines) are compared to existing data (measured, full symbols and extrapolated, empty symbols, all joined by dotted lines).

mentally, for these elements, the proton dripline has been determined $^{3}$ up to vanadium, with the last bound isotopes being ${ }^{35} \mathrm{~K},{ }^{35} \mathrm{Ca},{ }^{40} \mathrm{Sc},{ }^{40} \mathrm{Ti}$ and ${ }^{43} \mathrm{~V}$. For chromium, the last known isotope is ${ }^{43} \mathrm{Cr}$. Theoretical curves generally follow the experimental trends yielding an overall correct qualitative description of both $S_{1 \mathrm{p}}$ and $S_{2 \mathrm{p}}$. Looking more in detail, one observes that calculations tend to overestimate the measured separation energies in potassium and calcium, provide an excellent reproduction of scandium isotopes and underestimate titanium, vanadium and chromium. As a result, the position of the proton dripline is found at too small $N$ (with a difference of two or three neutrons) for the first two elements. In scandium, as well as vanadium, the dripline is correctly determined at $N=19$ and $N=20$ respectively. In titanium and chromium, it is also found respectively at $N=19$ and $N=20$, in this case one neutron away from what observed experimentally.

The cause of this small discrepancy can be traced back to the poor reproduction of the $Z=20$ gap by the $N N+3 N(\operatorname{lnl})$ Hamiltonian, as evident in Fig. 4. Here, two-proton separation energies are plotted as a function of proton number for different isotonic chains. One notices that, similarly to what observed in Fig. 2 for $N=20$, the $Z=20$ gap is overestimated by at least $5 \mathrm{MeV}$ in all considered isotones. The disagreement becomes more severe for low neutron numbers, which impacts the determination of the proton dripline in lighter isotopes. In spite of these shortcomings, this detailed analysis proves the overall remarkable quality of present ab initio calculations, not

\footnotetext{
3 Experimentally, the dripline is typically established by means of a void observation of one or several isotopes rather than by determining a negative value of $S_{1 \mathrm{p}}$ or $S_{2 \mathrm{p}}$.
} 


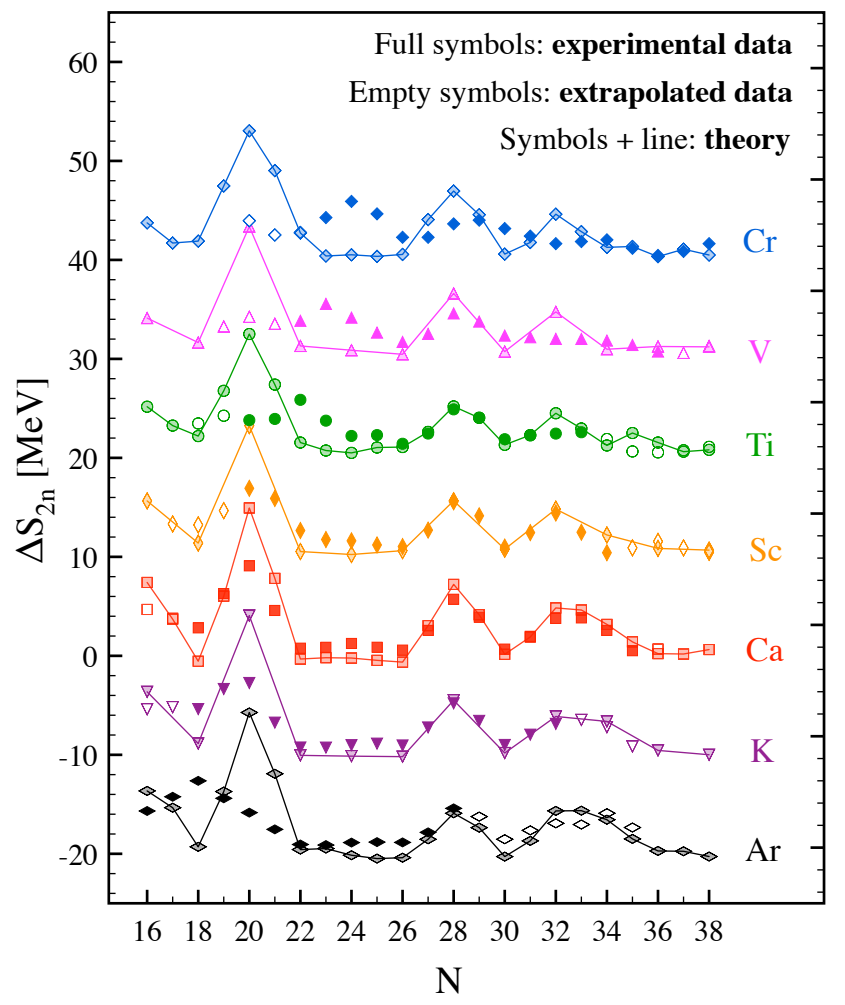

Fig. 5. Two-neutron shell gaps along $Z=18-24$ isotopic chains computed with the $N N+3 N(\operatorname{lnl})$ interaction (symbols joined by solid lines), compared to experimental (measured, full symbols and extrapolated, empty symbols) data. Both theoretical and experimental values are shifted by $(Z-20) \times 10$ $\mathrm{MeV}$ for a better readability.

dissimilar from what emerges from the systematic study reported in Ref. [51].

\subsection{Neutron gaps}

A finer insight regarding the magic character of specific neutron numbers can be gained by looking at so-called two-neutron shell gaps, defined as

$$
\Delta_{2 \mathrm{n}}(N, Z) \equiv S_{2 \mathrm{n}}(N, Z)-S_{2 \mathrm{n}}(N+2, Z)
$$

and displayed in Fig. 5. As for the $S_{2 \mathrm{n}}$, one first notices an overall excellent agreement with experiment, with the clear exception of the $N=20$ peak and its vicinity. While in semi-magic calcium isotopes calculations only fail to reproduce the height of the peak, experimental data for other isotopes show a displacement of the peak, linked to a possible disappearance of the $N=20$ magic number, which is not reproduced by the present calculations. In contrast, the $N=28$ peak is very well reproduced up to $Z=22$, with the description only slightly deteriorating for $Z=23$ and $Z=24$. The emergence of the $N=32$ subclosure is nicely visible in lighter elements, as well as the one at $N=34$ in argon, potassium and calcium. When going towards higher proton number their evolution is poorly

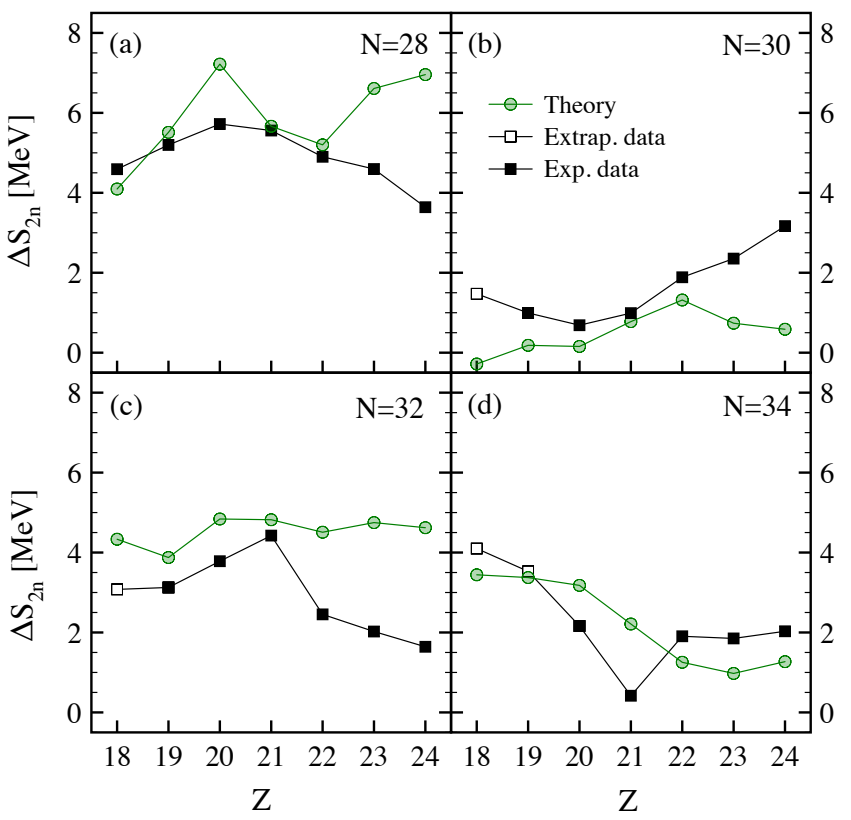

Fig. 6. Two-neutron shell gaps along four isotonic chains computed with the $N N+3 N(\operatorname{lnl})$ interaction (circles), compared to experimental (measured, full squares, and extrapolated, empty squares) data. Results for $N=28,30,32$ and 34 are shown in panels (a), (b), (c) and (d) respectively.

described starting with $N=34$ in scandium and $N=32$ in vanadium. The behaviour becomes even more inconsistent for chromium. Again, this might signal the importance of certain ingredients (e.g. quadrupole correlations) that are missing in the present theoretical framework.

In spite of these deficiencies, remarkably, all magic numbers as well as their qualitative evolution emerge "from first principles", i.e. starting solely from inter-nucleon interactions whose coupling constants have been adjusted only in few-body systems. Let us stress that, indeed, no ad hoc information about the magic character of these isotopes is inserted at any stage of the calculation. The emergence of this feature can be better appreciated in Fig. 6 where neutron gaps are compared to experimental (measured and extrapolated) data along $N=28,30,32$ and 34 isotonic chains. While there is room for improvement in $Z=22,23,24$ isotones for reasons discussed above, the overall description is very reasonable. In addition, calculations of the $N=28$ gaps were recently extended down to chlorine and sulfur [36] where an excellent agreement with novel precision mass measurement was also found.

\subsection{Three-point mass differences}

One of the longstanding challenges in low-energy nuclear physics relates to the microscopic description of nuclear superfluidity [52]. The microscopic origin of nucleonic pairing, i.e. how it originates in the context of a first-principle calculation and the role played by different types of manybody correlations, remains to be elucidated [53]. A fundamental, yet unresolved, question relates to how much 


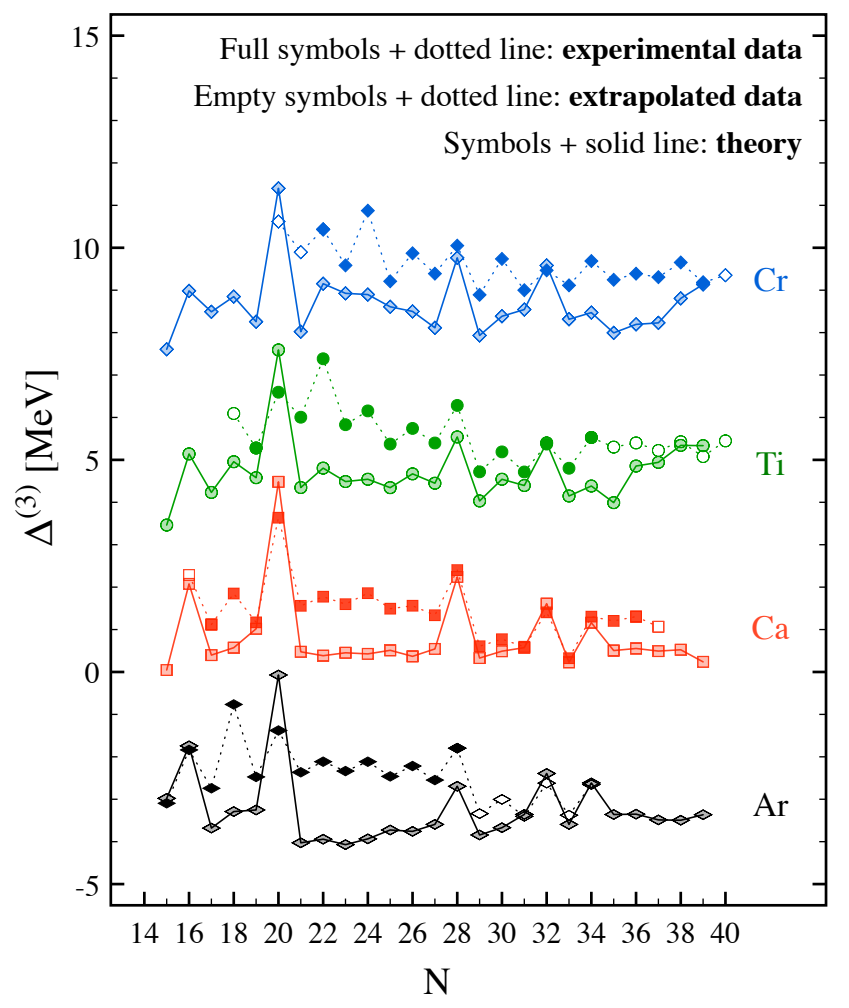

Fig. 7. Three-point mass differences along $Z=18,20,22$ and 24 isotopic chains computed with the $N N+3 N(\operatorname{lnl})$ interaction (symbols joined by solid lines), compared to experimental (measured, full symbols and extrapolated, empty symbols) data. Both calculated and experimental values are shifted by $(Z-20) \times 2 \mathrm{MeV}$ for a better readability.

of the pairing gap in finite nuclei is accounted for at lowest order [54, 55] and how much is due to higher-order processes, i.e. to the induced interaction associated with the exchange of collective medium fluctuations between paired particles $[56,57,58,59]$. By treating normal and anomalous propagators consistently and at the same level of approximation, GSCGF many-body scheme is in an excellent position to contribute to this quest. In finite nuclei, the odd-even mass staggering is a good measure of nucleonic, e.g. neutron, pairing. In particular, the three-point mass difference formula

$\Delta^{(3)}(N, Z) \equiv \frac{(-1)^{N}}{2}[E(N-1, Z)-2 E(N, Z)+E(N+1, Z)]$

successively evaluated for even and odd $N$ closely encompasses the pairing gap $[50,60]$ as long as $N$ does not correspond to a shell closure ${ }^{4}$. Calculated three-point mass differences for argon, calcium, titanium and chromium are compared to available experimental data in Fig. 7. In

\footnotetext{
${ }^{4}$ Note that $\Delta^{(3)}$ corresponds to half of the energy difference between the lowest unoccupied quasiparticle and the highest occupied quasihole states, that is the particle-hole neutron gap at the Fermi surface. At subshell closures, this is dominated by the gap among different nuclear orbits. However, for open neutron shells only the pairing contribution remains.
}

spite of a reasonable general trend, the pairing strength generated in the present ab initio calculations is too low compared to experiment. This feature is particularly visible for $N \in[21,27]$ isotopes in all considered chains, as well as beyond $N=34$ for calcium and titanium. Keeping in mind the possible deficiency of the currently used Hamiltonian, this result likely points to missing higher-order correlations $[56,57,58,59]$. The $\mathrm{ADC}(2)$ truncation scheme used here already includes both the lowest-order pairing term and the induced interaction resulting from the exchange of unperturbed particle-hole excitations. However, it does not account for collective vibrations. The extension of GSCGF to the $\mathrm{ADC}(3)$ level is envisaged in the near future, knowing that such a truncation does indeed seize important features of collective fluctuations and of their effect on superfluidity.

In titanium and chromium, theoretical and experimental three-point mass differences show further qualitative differences. In addition to the average value of $\Delta^{(3)}$ being too low, the increase of its oscillation between $N=20$ and $N=28$ compared to calcium isotopes along with the shellclosure disappearances at $N=28,32,34$ are not captured. The oscillation of $\Delta^{(3)}$ around its average is not related to the anomalous part of the self-energy (i.e. the pairing gap) but rather to its normal part (i.e. the effective meanfield) $[50,60]$. The qualitative evolution of this staggering from calcium to titanium and chromium pointed out above is thus a fingerprint of increased quadrupole correlations on the normal self-energy. The absence of this evolution in our theoretical calculation confirms the need to include these correlations consistently in both normal and anomalous channels. While extending GSCGF to the $\mathrm{ADC}(3)$ level should help better describing the staggering of $\Delta^{(3)}$, an explicit treatment of deformation will probably be the most

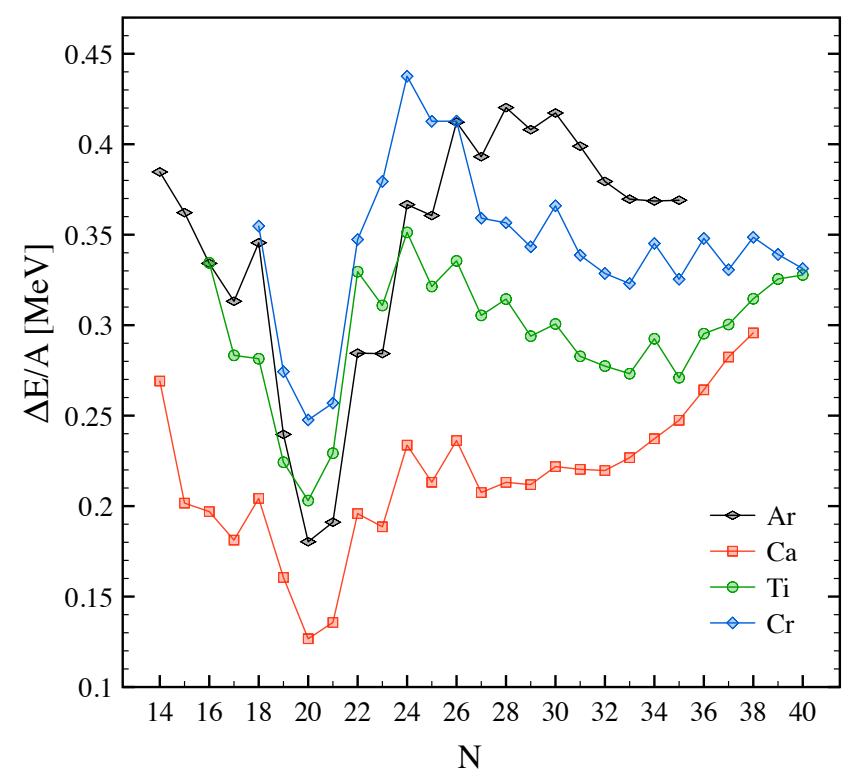

Fig. 8. Relative errors (theory - experiment) on total binding energies per nucleon along $Z=18,20,22$ and 24 isotopic chains. Both calculations and experimental data are taken from Fig. 1. 


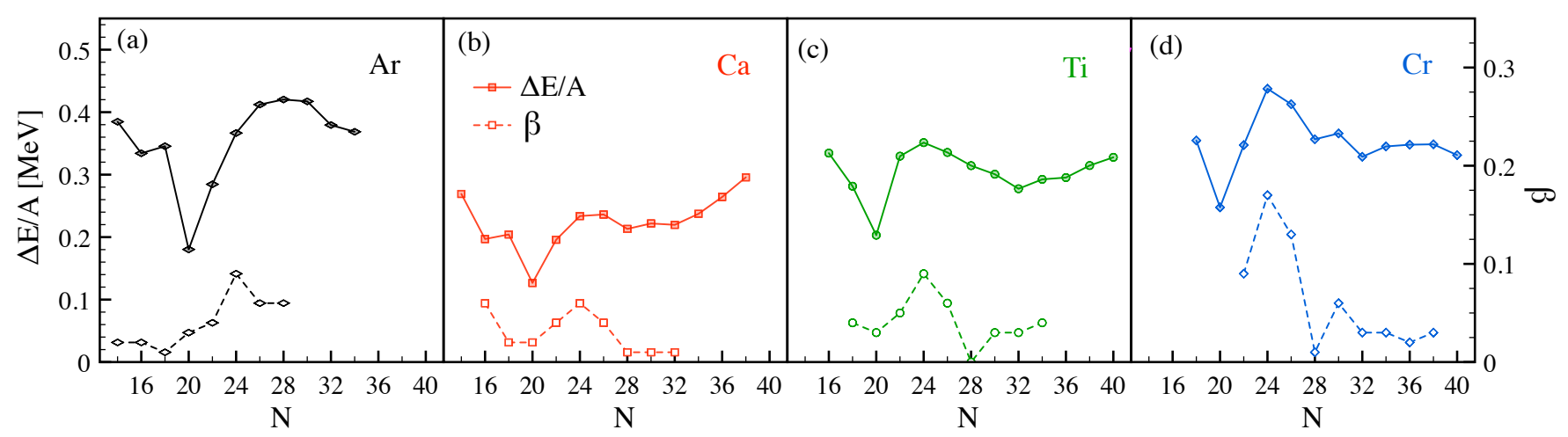

Fig. 9. Relative errors on total binding energies per nucleon for $Z=18,20,22$ and 24 isotopes (full symbols and solid lines, taken from Fig. 8) and corresponding deformation parameter $\beta$ computed via HFB calculations with the Skyrme SLy 4 interaction [61] (empty symbols and dashed lines).

efficient way to reach a quantitative agreement whenever quadrupole fluctuations become truly collective, i.e. as one moves significantly away from semi-magic systems.

\subsection{Effects of deformation}

For several of the quantities discussed above, the poorer agreement with theoretical data when departing from semimagic calcium has been ascribed to an inefficient description of quadrupole correlations. To substantiate this observation, differences between computed and experimental ground-state energies per nucleon are displayed in Fig. 8 for four isotopic chains. Unsurprisingly, the best agreement with experimental values is found for calcium isotopes. For this chain, the error is dominated by the many-body truncation at $\operatorname{ADC}(2)$ and by possible flaws of the adopted nuclear Hamiltonian. Other chains perform generally worse, with the quality of the description deteriorating in particular for neutron-rich argon and chromium isotopes. In all cases a clear minimum is visible at $N=20$ and a maximum around $N=24$, which suggests a correlation with the closed- or open-shell character of the neutrons and the associated absence or presence of static deformation.

This hypothesis is examined in Fig. 9, where the four curves of Fig. 8 are plotted separately and compared to the deformation parameter $\beta$ obtained in (single-reference) energy density functional calculations [61]. The correlation between the two quantities is striking for all chains. This observation supports the intuition that the collective quadrupole correlations arising in doubly-open shell systems can hardly be captured by present SU(2)-conserving calculations.

Even if in principle all correlations can be accounted for in the current theoretical scheme, one would need to include very high orders in the expansion in order to grasp such quadrupole static correlations. Indeed, these are typically associated with the coherent superposition of many particle-many hole excitations that are not included in the low-order many-body truncation schemes currently at reach. Extending beyond the $\mathrm{ADC}(3)$ approximation involves a factorial increase in the numbers of diagrams and would need a shift of paradigm in which all contributions are dealt with at once through stochastic sampling [62]. An alternative solution is the extension of existing expansion methods towards SU(2)-breaking schemes that will enable an efficient description of static deformation from the outset.

\section{Radii}

Among the basic nuclear properties addressed by ab initio calculations in the past few years, the size of medium-mass nuclei has typically represented (and, to a good extent, still represents) one of the main challenges. The first sets of calculations that successfully reproduced ground-state energies of oxygen isotopes failed to provide, at the same time, a good description of charge radii [33]. The $\mathrm{NNLO}_{\text {sat }}$ Hamiltonian, specifically introduced to cure this issue [37], very much improved the description of radii although discrepancies for neutron-rich systems have been shown to persist $[33,63]$. An unsatisfactory account of nuclear sizes remains for several Hamiltonians that are currently employed in state-of-the-art calculations [64, 8]. Very recently, new generations of chiral interactions have been proposed and shown to provide promising results for charge radii of closed-shell [65] as well as some open-shell [30] mediummass nuclei. The behaviour along isotopic chains around calcium remains however to be investigated. In Ref. [8] charge radii of oxygen, calcium and nickel isotopes have been systematically investigated with the $N N+3 N(\operatorname{lnl})$ and $\mathrm{NNLO}_{\text {sat }}$ Hamiltonians. The study confirmed the good performance of $\mathrm{NNLO}_{\text {sat }}$ up to the nickel chains. Here, in addition to a more refined analysis of calcium isotopes, charge radii along argon, titanium and chromium chains are presented.

Mean square (m.s.) charge radii are computed starting from m.s. point-proton radii $\left\langle r_{\mathrm{p}}^{2}\right\rangle$ as follows

$$
\left\langle r_{\mathrm{ch}}^{2}\right\rangle=\left\langle r_{\mathrm{p}}^{2}\right\rangle+\left\langle R_{\mathrm{p}}^{2}\right\rangle+\frac{N}{Z}\left\langle R_{\mathrm{n}}^{2}\right\rangle+\frac{3 \hbar^{2}}{4 m_{\mathrm{p}}^{2} c^{2}} .
$$

The last term corresponds to the relativistic Darwin-Foldy correction [66] amounting to $0.033 \mathrm{fm}^{2} .\left\langle R_{\mathrm{p}}^{2}\right\rangle$ and $\left\langle R_{\mathrm{n}}^{2}\right\rangle$ 


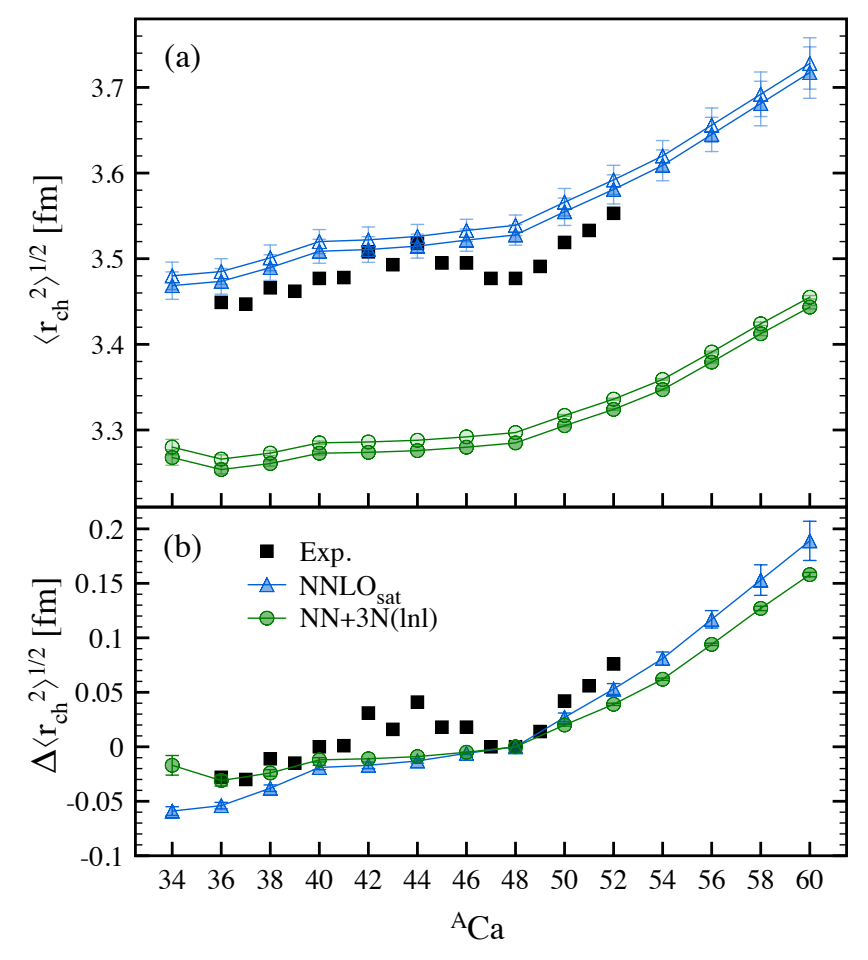

Fig. 10. (a) Absolute root mean square charge radii of calcium isotopes and (b) differential ones relative to ${ }^{48} \mathrm{Ca}$ computed with the $N N+3 N(\operatorname{lnl})$ and $\mathrm{NNLO}_{\text {sat }}$ interactions. Available experimental data from Refs. [67, 63, 71] are displayed. Dark (light) symbols were obtained using a value $\left\langle R_{\mathrm{p}}^{2}\right\rangle=0.6905 \mathrm{fm}^{2}[72]$ $\left(\left\langle R_{\mathrm{p}}^{2}\right\rangle=0.770 \mathrm{fm}^{2}[68]\right)$ in Eq. (7). Error bars account for the uncertainty associated to model space truncation (see text for details).

represent the m.s. charge radius of the proton and the neutron respectively. While the latter is relatively well established, $\left\langle R_{\mathrm{n}}^{2}\right\rangle=-0.1149(27) \mathrm{fm}^{2}$ [67], the determination of the former has been debated and revised in the past few years. In the past, the value of $\left\langle R_{\mathrm{p}}^{2}\right\rangle \simeq 0.77 \mathrm{fm}^{2}$ inferred from electron scattering experiments was commonly used and included in the CODATA compilation [68]. Recent experiments, including electronic and muonic hydrogen Lamb shift measurements, favour a lower m.s. radius of $\left\langle R_{\mathrm{p}}^{2}\right\rangle \simeq 0.70 \mathrm{fm}^{2}$ [69]. As a result, the CODATA value was updated to $\left\langle R_{\mathrm{p}}^{2}\right\rangle \simeq 0.7079 \mathrm{fm}^{2}$ [70]. This value is adopted in the present work and used in Eq. (7), unless specified otherwise. Given the large variation of $\left\langle R_{\mathrm{p}}^{2}\right\rangle$ found in the literature, however, it is worth investigating its impact on computed charge radii, specially in comparison with other sources of theoretical error in the calculation.

Figure 10 shows root m.s. (r.m.s.) charge radii along calcium isotopes computed with the $N N+3 N(\operatorname{lnl})$ and $\mathrm{NNLO}_{\text {sat }}$ Hamiltonians, either as absolute, panel (a), or relative to ${ }^{48} \mathrm{Ca}$, panel (b). For each interaction, the two sets of points (dark and light symbols) were obtained with two different values of the proton radius in Eq. (7), respectively $\left\langle R_{\mathrm{p}}^{2}\right\rangle=0.6905 \mathrm{fm}^{2}[72]$ and $\left\langle R_{\mathrm{p}}^{2}\right\rangle=0.770 \mathrm{fm}^{2}$ [68]. The two values are representative of the two sets of experimental results discussed above. For each set of points, error bars conservatively account for the uncertainty coming from truncation of the one-body basis in the calculation. Specifically, they are obtained from the variation associated to different values of the HO frequency $\hbar \omega$ around the optimal value (itself determined as the closest point to the intersection of the different $e_{\max }$ curves, see Fig. 5 of Ref. [8]). While such variation is sizeable for $\mathrm{NNLO}_{\text {sat }}$, it is generally smaller than the size of the points for $N N+3 N(\operatorname{lnl})$. In Ref. [8] the effects of higher-order (specifically, ADC(3)) correlations on charge radii was also assessed for selected doubly-closed isotopes. For both interactions, the associated uncertainties were of the order of $1 \%$, i.e. they are comparable to the ones coming from model-space truncation in the case of $\mathrm{NNLO}_{\text {sat }}$. Hence, one should virtually add such error bars also to the $N N+3 N(\operatorname{lnl})$ results. For the latter, another possible source of error comes from neglecting many-body radius operators induced by the SRG evolution. However, recent calculations performed with a similar interaction have shown that the consistent inclusion of such operators does not impact significantly the final result [73]. In conclusion, for absolute r.m.s. charge radii, the chosen value of $\left\langle R_{\mathrm{p}}^{2}\right\rangle$ can lead to a $0.5 \%$ variation, whereas uncertainties associated to model-space and manybody truncations are each of the order of $1 \%$, with the caveat that many-body truncations have been estimated only on closed-shell (not deformed) isotopes. The situation is even more favourable for differential radii, as visible in Fig. 10(b). Here most of the errors cancel out and one is left with some sizeable model-space truncation uncertainty only for the most neutron-rich isotopes. These improved calculations do not present significant differences with respect to the ones discussed in Ref. [8]. Results obtained with $N N+3 N(\operatorname{lnl})$ underestimate the experimental values by about $5 \%$ throughout the calcium chain. Although the main experimental trend is roughly captured by the theoretical curves (see also Fig. 12 and associated discussion), two of its peculiar features, namely the parabolic behaviour between ${ }^{40} \mathrm{Ca}$ and ${ }^{48} \mathrm{Ca}$ and the steep rise beyond ${ }^{48} \mathrm{Ca}$, are missing.

Let us now move to results for argon, titanium and chromium isotopes, displayed in Fig. 11. Globally, the behaviour is similar to the one observed in the calcium chain, with $\mathrm{NNLO}_{\text {sat }}$ calculations very close to experimental data and $N N+3 N(\operatorname{lnl})$ underestimating experiment by about 5 to $10 \%$. In argon, see Fig. 11 (a), charge radii computed with $\mathrm{NNLO}_{\text {sat }}$ reproduce very well existing data, with the notable exception of the most neutron-rich isotope available, ${ }^{46} \mathrm{Ar}$. The trend presents a kink at this nucleus, after which a steady increase with neutron number is observed until $N=34$ where a second kink appears. Results obtained with $N N+3 N(\operatorname{lnl})$ follow a similar behaviour past ${ }^{46} \mathrm{Ar}$, as one can appreciate by looking at relative charge radii displayed in Fig. 11 (d). Below $N=28$, however, the $N N+3 N(\operatorname{lnl})$ slope is somehow different from $\mathrm{NNLO}_{\text {sat }}$ and experimental data. Experimental points are more scarce for titanium and chromium, with essentially only stable or long-lived isotopes available. In titanium, Fig. 11 (b) and (e), isotopes with $N=22-26$ are well reproduced by $\mathrm{NNLO}_{\text {sat }}$, while ${ }^{50} \mathrm{Ti}$ is overestimated, simi- 


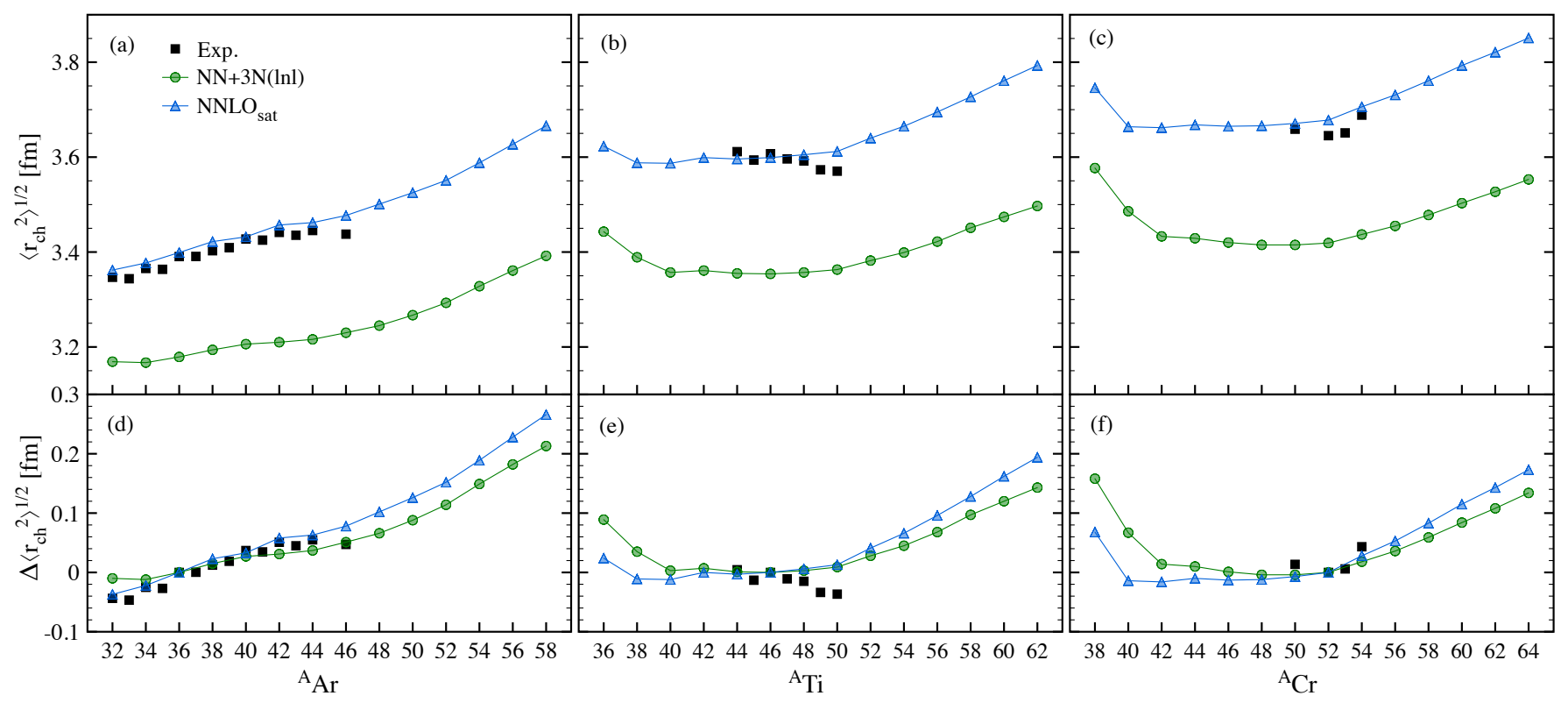

Fig. 11. Root mean square charge radii of (a) argon, (b) titanium and (c) chromium isotopes computed with the $N N+3 N(\operatorname{lnl})$ and $\mathrm{NNLO}_{\text {sat }}$ interactions. Experimental data are taken from Ref. [67]. Panels (d), (e) and (f) show corresponding differential radii relative to ${ }^{36} \mathrm{Ar},{ }^{46} \mathrm{Ti}$ and ${ }^{52} \mathrm{Cr}$ respectively.

larly to ${ }^{46} \mathrm{Ar} . N N+3 N(\operatorname{lnl})$ follows the same relative trend around stability, with slightly different slopes in the protonand neutron-rich regions. Analogous behaviour is observed for chromium, shown in Fig. 11 (c) and (f). Also in this case the radius of the $N=28$ isotope, ${ }^{52} \mathrm{Cr}$, is overestimated by $\mathrm{NNLO}_{\text {sat }}$ calculations, which instead give an excellent reproduction of neighbouring ${ }^{50} \mathrm{Cr}$ and ${ }^{54} \mathrm{Cr}$. Curves obtained with $N N+3 N(\operatorname{lnl})$ present the same general features as in the titanium chain.

To better gauge the overall quality of the theoretical description, m.s. charge radii along all four isotopic chains are shown in Fig. 12. By examining available experimental data, one can identify three distinct regions ${ }^{5}$ :

a) Below $N=20$, a steady increase with mild odd-even staggering is observed for calcium and argon.

b) Between $N=20$ and $N=28$, the slope of the experimental trend changes noticeably, going from positive (argon) to null (calcium) and negative (titanium and chromium). Moreover, this is superposed with an inverse parabolic behaviour characterised by a marked odd-even staggering. The parabolic trend is weak in argon and titanium, but pronounced in calcium.

c) Above $N=28$, one finds a steep increase with small or even absent signs of odd-even staggering and shell closures.

Computed charge radii do reproduce some but not all of these experimental trends. Below $N=20$, the steady behaviour is captured by the calculations, although a slight shift is present for calcium. In the central region, the change

\footnotetext{
${ }^{5}$ Notice that this differentiation also applies to odd- $Z$ chains
} around calcium and extends up to iron, see Ref. [74].

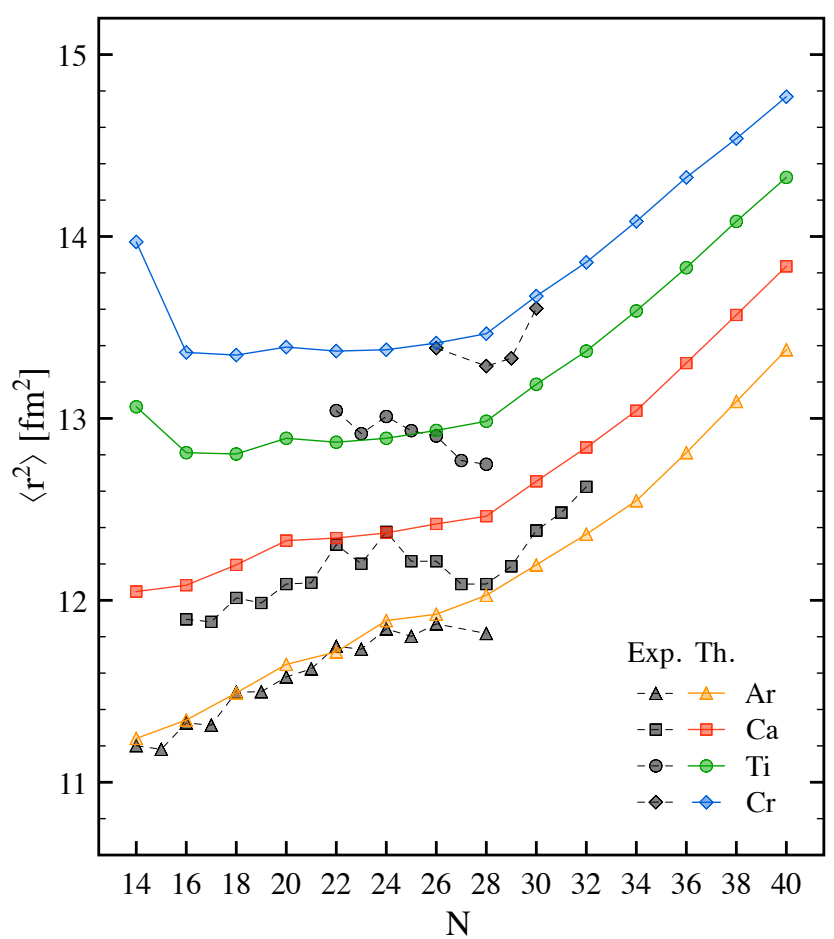

Fig. 12. Mean square charge radii of argon, calcium, titanium and chromium isotopes computed with the $\mathrm{NNLO}_{\text {sat }}$ interaction (coloured symbols and solid lines) compared to available experimental data $[67,63,71]$ (dark grey symbols and dashed lines). 


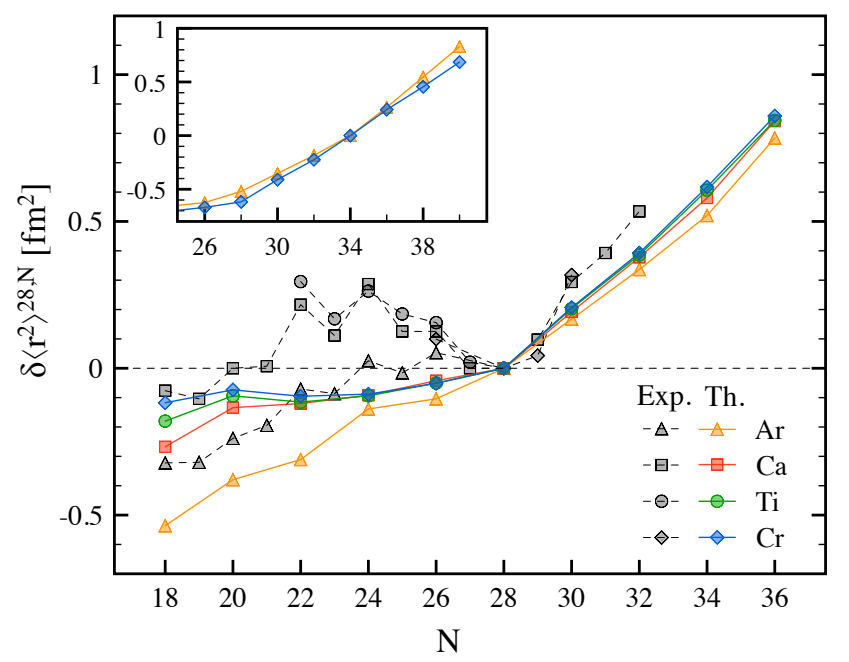

Fig. 13. Changes in m.s. charge radii for argon, calcium, titanium and chromium relative to $N=28$. Results obtained with the $\mathrm{NNLO}_{\text {sat }}$ Hamiltonian (coloured symbols and solid lines) are compared to existing experimental data [67,63, 71] (grey symbols and dashed lines). In the inset, changes in m.s. charge radii relative to $N=34$ are shown for argon and chromium isotopes.

in slope from argon to chromium is qualitatively reproduced. In contrast, the parabolic behaviour is basically absent in all calculated curves. We note that the charge radii for calcium between $N=20-28$ have been explained in terms of coupling to collective modes in Ref. [75] and excitations across the $s d$ and $p f$ orbits using the shell model approach [76]. In both cases, quadrupole excitations to (possibly deformed) states are involved. The particlevibration coupling at the origin of this mechanism is encoded in the $\mathrm{ADC}(3)$ many-body truncation and in its twin approach, the Faddeev Random phase approximation [77], which is slightly more sophisticated for collective modes. Thus, $\mathrm{ADC}(3)$ stands out as the minimum requirement to be able to reproduce the inverted bell behaviour of radii in the central region. However, the above early studies were based on phenomenological interactions. For ab initio applications, it is not clear a priori to what extent the $\operatorname{ADC}(3)$ will be sufficient to resolve the low-energy quadrupole deformations with current soft chiral Hamiltonians.

For all isotopes, the theoretical charge radius at $N=28$ is systematically larger than the measured one. This also affects the slope beyond this point, which results less steep than what observed in experimental data. This inability to reproduce the pronounced kink at $N=28$ is common to other ab initio calculations as initially discussed in Ref. [63]. In order to analyse this feature in more details, Fig. 13 shows measured and computed m.s. charge radii relative to $N=28$. The two experimental curves extending beyond $N=28$ do indeed present the same rise towards $N=30$. Manganese $(Z=25)$ and iron $(Z=26)$, for which experimental data are available, also follow this trend. The same behaviour, with a kink followed by a steep rise essentially independent of $Z$, is found at the

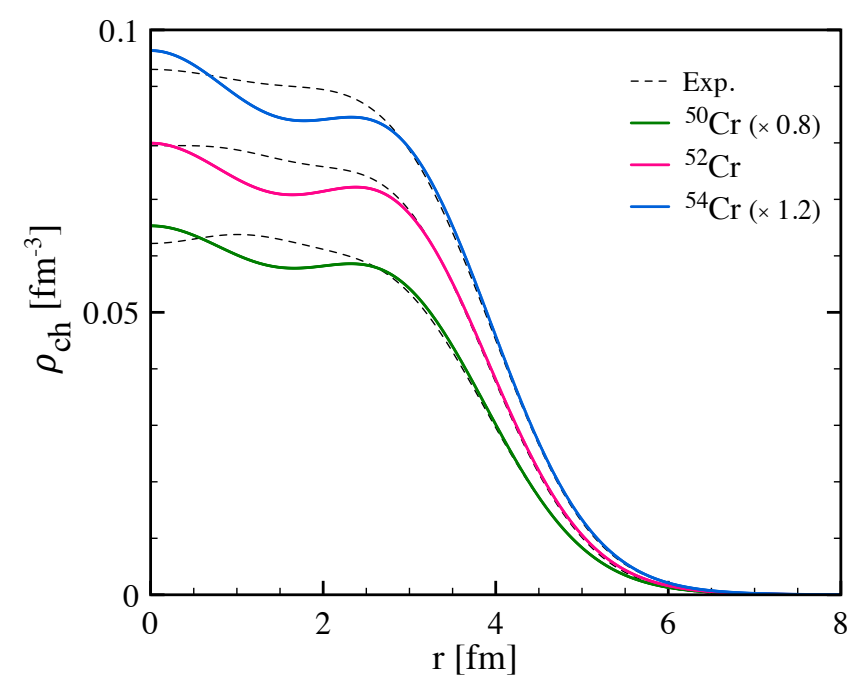

Fig. 14. Charge density distributions of three chromium isotopes. $\mathrm{NNLO}_{\text {sat }}$ calculations are compared to density profiles determined via electron scattering [80]. Curves relative to ${ }^{50} \mathrm{Cr}$ and ${ }^{54} \mathrm{Cr}$ have been rescaled for better readability.

$N=50$ and $N=82$ magic numbers [74]. Remarkably, the theoretical curves capture this basic feature, yielding radii that increase almost independently of $Z$ beyond $N=28$. As already remarked, however, the slope is less steep than the experimental one, which represents a challenge for most of nuclear structure calculations. Interestingly, a second, less pronounced kink is visible at $N=34$ (see inset of Fig. 13), suggesting the presence of a weak shell closure. A similar feature is observed in the charge radii computed with the $N N+3 N(\operatorname{lnl})$ Hamiltonian, see Figs. 10 and 11 . For both interactions, the angle of the kink decreases smoothly with $Z$, which is consistent with the evolution of the $N=34$ neutron gaps computed with $N N+3 N(\operatorname{lnl})$ and reported in Fig. 6(d).

To conclude the present section, some examples of charge density distributions in chromium isotopes are shown in Fig. 14. Theoretically, the charge distribution is computed starting from the point-proton distribution and folding it with the charge distribution of the proton, as detailed in Ref. [78]. Corrections associated with the center of mass motion in the $\mathrm{HO}$ basis are seen to be negligible for masses above $A=16$ [79]. In Fig. 14 distributions of ${ }^{50,52,54} \mathrm{Cr}$ computed with $\mathrm{NNLO}_{\text {sat }}$ are compared to charge profiles determined from electron scattering cross sections [80]. Theoretical distributions follow closely the experimental curves in the region around and above $r_{\mathrm{ch}}$. In contrast, for all three isotopes the behaviour differ in the nuclear interior, with the calculations displaying a dip around $1.5 \mathrm{fm}$ that is not present, or not probed, in the experimental distributions. One does not observe a qualitatively different behaviour for ${ }^{52} \mathrm{Cr}$, whose value of $r_{\text {ch }}$ slightly departs from experiment. A similar level of agreement between SCGF calculations and experiment was recently found for argon and calcium isotopes [35] and for ${ }^{132} \mathrm{Sn}$, although theoretical error bars were lager for the latter case [11]. 


\section{Conclusions}

Correlation expansion methods represent a promising longterm option to simulate the majority, if not all, of atomic nuclei from first principles. To this purpose, the choice of the reference state, including the use of deformed basis states and the possibility of breaking symmetries, is crucial, notably to account for essential static correlations from the outset. So far, ab initio approaches have mainly exploited the breaking of $\mathrm{U}(1)$ symmetry associated to particle number conservation to account for static pairing correlations. In the past few years, this strategy has enabled computations of semi-magic, i.e. singly open-shell nuclei, where quadrupole correlations associated to nuclear deformation are typically weak, i.e. predominantly dynamical. In the present work such U(1)-breaking, SU(2)conserving calculations are pushed away from semi-magic nuclei in a systematic fashion for the first time. Results are overall encouraging, with many general experimental features captured by the ab initio simulations. At the same time, a degradation of the description for certain groups of nuclei signals the inefficient account of (static) quadrupole correlations and calls for a $\mathrm{SU}(2)$-breaking extension of the present theoretical framework.

Specifically, bulk nuclear properties (ground-state energies, charge radii and density distributions) were computed along seven isotopic chains around calcium, from argon to chromium. Calculations were performed within the Gorkov self-consistent Green's function approach at second order and employed two state-of-the-art two- plus three-nucleon Hamiltonians, $N N+3 N(\operatorname{lnl})$ and $\mathrm{NNLO}_{\text {sat }}$. $N N+3 N(\operatorname{lnl})$ results provide a good global description of ground-state energies. Total energies are slightly underestimated, consistently with missing higher-order correlations as discussed in detail in Ref. [8]. Differential energies, i.e. one- and two-nucleon separation energies as well as twoneutron shell gaps, are generally in excellent agreement with experiment. In particular, neutron magic numbers $N=28,32,34$ emerge and evolve following experimental trends. The largest discrepancy with experimental data is found for the $N, Z=20$ gaps, both overestimated by the calculations. This impacts the description of the proton dripline, which however remains reasonably reproduced. In contrast, three-point mass differences along the various isotopic chains evidence that present calculations do not provide sufficient pairing strength. The future inclusion of higher-order, e.g. $\mathrm{ADC}(3)$, corrections accounting for collective fluctuations might result instrumental for a more accurate description of pairing properties. While such computations are routine in Dyson-SCGF (i.e., for closed shells), they become computationally challenging in the Gorkov formalism due to the increase in the number of Bogolyubov mean-field orbits resulting from $\mathrm{SU}(1)$-breaking. A full Gorkov-ADC(3) will require improved algorithms, such as importance truncation, but it is within reach and can be implemented in the foreseeable future.

As remarked in Ref. [8], $N N+3 N(\operatorname{lnl})$ calculations yield charge radii that underestimate the experimental measurements by about 5 to $10 \%$ throughout all considered chains. Still, relative trends are generally good, which points to some small systematic deficiencies in the Hamiltonian. In contrast, $\mathrm{NNLO}_{\text {sat }}$ provides an overall good reproduction of both absolute and relative charge radii. The main experimental trends below $N=20$, between $N=20$ and $N=28$ and above $N=28$ are qualitatively described. The largest discrepancy with data is detected for $N=28$ isotopes, whose radius is overestimated in all considered elements. As a consequence, the steep rise past $N=28$ observed in calcium and chromium is not reproduced to a full extent by the present calculations. The inability to correctly describe the charge radius difference between ${ }^{48} \mathrm{Ca}$ and ${ }^{52} \mathrm{Ca}$ is common to nearly all existing nuclear structure calculations (with the notable exception of Ref. [81]) and currently represents a challenge in particular for ab initio approaches.

For some of the doubly open-shell nuclei considered in this study, strong (i.e. static) quadrupole correlations are expected to play an important role and lead to the onset of deformation. Indeed, a careful comparison between computed and experimental ground-state energies reveals a remarkable correlation between the error with respect to experiment and the expected degree of deformation (quantified through the deformation parameter $\beta$ obtained via EDF calculations [61]). Such correlations are likely to impact the calculated observables but can be hardly accounted for in the current scheme that uses (rotational) symmetry-conserving reference states and incremental extensions of the formalism. In fact, any increase in the $\operatorname{ADC}(n)$ order beyond $n=3$ would be impractical due to the factorial increase in diagrams and degrees of freedom. Besides, such a truncation scheme is unlikely to resolve deformation degrees of freedom until several orders beyond the current capabilities. To break through these limitations different approaches could be envisaged, such as the stochastic sampling of the self-energy or a $\mathrm{SU}(2)$ breaking scheme. In the first case, one would still work in a standard (spherical or partially deformed) basis but diagrams are summed to very high orders using bold diagrammatic Monte Carlo techniques [62]. This approach is particularly suited to address correlations at medium energies that have been identified as key ingredients to devise ab initio nucleon-nucleus optical potentials [82]. In the second path, the extension towards a $\mathrm{SU}(2)$-breaking scheme would impose nuclear deformation already at the level of the reference state and allow many-body truncations at low $\operatorname{ADC}(n)$ orders, still requiring a final projection on good angular momentum. Both approaches will involve sophisticated extensions of the SCGF formalism and will be long-term developments.

\section{Acknowledgements}

The authors wish to thank R. Garcia Ruiz and F. Raimondi for useful discussions. Calculations were performed by using HPC resources from GENCI-TGCC (Contracts No. A005057392, A007057392) and at the DiRAC Complexity system at the University of Leicester (BIS National Einfrastructure capital grant No. ST/K000373/1 and STFC grant No. ST/K0003259/1). This work was supported by 
the United Kingdom Science and Technology Facilities Council (STFC) under Grant No. ST/L005816/1 and in part by the NSERC Grant No. SAPIN-2016-00033. TRIUMF receives federal funding via a contribution agreement with the National Research Council of Canada.

\section{References}

1. W. H. Dickhoff and C. Barbieri, Prog. Part. Nucl. Phys. 52, 377 (2004).

2. K. Kowalski, D. J. Dean, M. Hjorth-Jensen, T. Papenbrock, and P. Piecuch, Phys. Rev. Lett. 92, 132501 (2004).

3. S. K. Bogner, R. J. Furnstahl, and A. Schwenk, Prog. Part. Nucl. Phys. 65, 94 (2010).

4. S. Binder, J. Langhammer, A. Calci, and R. Roth, Physics Letters B 736, 119 (2014).

5. H. Hergert, S. K. Bogner, T. D. Morris, S. Binder, A. Calci, J. Langhammer, and R. Roth, Phys. Rev. C 90, 041302 (2014).

6. G. Hagen, G. R. Jansen, and T. Papenbrock, Phys. Rev. Lett. 117, 172501 (2016).

7. R. Taniuchi et al., Nature 569, 53 (2019).

8. V. Somà, P. Navrátil, F. Raimondi, C. Barbieri, and T. Duguet, Phys. Rev. C 101, 014318 (2020).

9. T. D. Morris, J. Simonis, S. R. Stroberg, C. Stumpf, G. Hagen, J. D. Holt, G. R. Jansen, T. Papenbrock, R. Roth, and A. Schwenk, Phys. Rev. Lett. 120, 152503 (2018).

10. P. Gysbers et al., Nature Phys. 15, 428 (2019).

11. P. Arthuis, C. Barbieri, M. Vorabbi, and P. Finelli, (2020), arXiv:2002.02214 [nucl-th] .

12. Z. Rolik, A. Szabados, and P. R. Surján, The Journal of Chemical Physics 119, 1922 (2003).

13. P. R. Surján, Z. Rolik, A. Szabados, and D. Köhalmi, Annalen der Physik 13, 223 (2004).

14. B. R. Barrett, P. Navratil, and J. P. Vary, Prog. Part. Nucl. Phys. 69, 131 (2013).

15. E. Gebrerufael, K. Vobig, H. Hergert, and R. Roth, Phys. Rev. Lett. 118, 152503 (2017).

16. H. Hergert, Phys. Scripta 92, 023002 (2017).

17. A. Tichai, E. Gebrerufael, K. Vobig, and R. Roth, Phys. Lett. B 786, 448 (2018).

18. S. K. Bogner, H. Hergert, J. D. Holt, A. Schwenk, S. Binder, A. Calci, J. Langhammer, and R. Roth, Phys. Rev. Lett. 113, 142501 (2014).

19. G. R. Jansen, J. Engel, G. Hagen, P. Navratil, and A. Signoracci, Phys. Rev. Lett. 113, 142502 (2014).

20. S. R. Stroberg, S. K. Bogner, H. Hergert, and J. D. Holt, Ann. Rev. Nucl. Part. Sci. 69, 307 (2019).

21. T. Duguet, Journal of Physics G: Nuclear and Particle Physics 42, 025107 (2014).

22. T. Duguet and A. Signoracci, J. Phys. G 44, 015103 (2017).

23. Y. Qiu, T. M. Henderson, T. Duguet, and G. E. Scuseria, Phys. Rev. C 99, 044301 (2019).

24. M. Bender, P.-H. Heenen, and P.-G. Reinhard, Rev. Mod. Phys. 75, 121 (2003).
25. V. Somà, T. Duguet, and C. Barbieri, Phys. Rev. C 84, 064317 (2011).

26. A. Signoracci, T. Duguet, G. Hagen, and G. R. Jansen, Phys. Rev. C 91, 064320 (2015).

27. A. Tichai, P. Arthuis, T. Duguet, H. Hergert, V. Somà, and R. Roth, Phys. Lett. B 786, 195 (2018).

28. A. Tichai, R. Roth, and T. Duguet, Front. in Phys. 8, $164(2020)$.

29. J. M. Yao, B. Bally, J. Engel, R. Wirth, T. R. Rodríguez, and H. Hergert, Phys. Rev. Lett. 124, 232501 (2020).

30. S. Novario, G. Hagen, G. Jansen, and T. Papenbrock, (2020), arXiv:2007.06684 [nucl-th] .

31. V. Somà, C. Barbieri, and T. Duguet, Phys. Rev. C 87, 011303 (2013).

32. V. Somà, A. Cipollone, C. Barbieri, P. Navrátil, and T. Duguet, Phys. Rev. C 89, 061301 (2014).

33. V. Lapoux, V. Somà, C. Barbieri, H. Hergert, J. D. Holt, and S. R. Stroberg, Phys. Rev. Lett. 117, 052501 (2016).

34. E. Leistenschneider, M. P. Reiter, S. Ayet San Andrés, B. Kootte, J. D. Holt, P. Navrátil, C. Babcock, C. Barbieri, B. R. Barquest, J. Bergmann, J. Bollig, T. Brunner, E. Dunling, A. Finlay, H. Geissel, L. Graham, F. Greiner, H. Hergert, C. Hornung, C. Jesch, R. Klawitter, Y. Lan, D. Lascar, K. G. Leach, W. Lippert, J. E. McKay, S. F. Paul, A. Schwenk, D. Short, J. Simonis, V. Somà, R. Steinbrügge, S. R. Stroberg, R. Thompson, M. E. Wieser, C. Will, M. Yavor, C. Andreoiu, T. Dickel, I. Dillmann, G. Gwinner, W. R. Plaß, C. Scheidenberger, A. A. Kwiatkowski, and J. Dilling, Phys. Rev. Lett. 120, 062503 (2018).

35. C. Barbieri, N. Rocco, and V. Somà, Phys. Rev. C 100, 062501 (2019).

36. M. Mougeot, D. Atanasov, C. Barbieri, K. Blaum, M. Breitenfeld, A. de Roubin, T. Duguet, S. George, F. Herfurth, A. Herlert, J. D. Holt, J. Karthein, D. Lunney, V. Manea, P. Navrátil, D. Neidherr, M. Rosenbusch, L. Schweikhard, A. Schwenk, V. Somà, A. Welker, F. Wienholtz, R. N. Wolf, and K. Zuber, Phys. Rev. C 102, 014301 (2020).

37. A. Ekström, G. R. Jansen, K. A. Wendt, G. Hagen, T. Papenbrock, B. D. Carlsson, C. Forssén, M. HjorthJensen, P. Navrátil, and W. Nazarewicz, Phys. Rev. C 91, 051301 (2015).

38. V. Somà, C. Barbieri, and T. Duguet, Phys. Rev. C 89, 024323 (2014).

39. D. R. Entem and R. Machleidt, Phys. Rev. C 68, 041001 (2003).

40. R. Machleidt and D. Entem, Physics Reports 503, 1 (2011).

41. A. Carbone, A. Cipollone, C. Barbieri, A. Rios, and A. Polls, Phys. Rev. C 88, 054326 (2013).

42. A. Cipollone, C. Barbieri, and P. Navrátil, Phys. Rev. C 92, 014306 (2015).

43. A. Cipollone, C. Barbieri, and P. Navrátil, Phys. Rev. Lett. 111, 062501 (2013).

44. C. Barbieri, Journal of Physics: Conference Series 529, 012005 (2014). 
45. F. Raimondi and C. Barbieri, Phys. Rev. C 97, 054308 (2018).

46. J. Ripoche, A. Tichai, and T. Duguet, Eur. Phys. J. A 56, 40 (2020).

47. W. Huang, G. Audi, M. Wang, F. G. Kondev, S. Naimi, and X. Xu, Chinese Physics C 41, 030002 (2017).

48. S. Michimasa, M. Kobayashi, Y. Kiyokawa, S. Ota, D. S. Ahn, H. Baba, G. P. A. Berg, M. Dozono, N. Fukuda, T. Furuno, E. Ideguchi, N. Inabe, T. Kawabata, S. Kawase, K. Kisamori, K. Kobayashi, T. Kubo, Y. Kubota, C. S. Lee, M. Matsushita, H. Miya, A. Mizukami, H. Nagakura, D. Nishimura, H. Oikawa, H. Sakai, Y. Shimizu, A. Stolz, H. Suzuki, M. Takaki, H. Takeda, S. Takeuchi, H. Tokieda, T. Uesaka, K. Yako, Y. Yamaguchi, Y. Yanagisawa, R. Yokoyama, K. Yoshida, and S. Shimoura, Phys. Rev. Lett. 121, 022506 (2018).

49. X. Xu et al., Phys. Rev. C 99, 064303 (2019), arXiv:1905.12577 [nucl-ex] .

50. T. Duguet, P. Bonche, P.-H. Heenen, and J. Meyer, Phys. Rev. C 65, 014311 (2001).

51. J. Holt, S. Stroberg, A. Schwenk, and J. Simonis, (2019), arXiv:1905.10475 [nucl-th] .

52. D. J. Dean and M. Hjorth-Jensen, Rev. Mod. Phys. 75, 607 (2003).

53. T. Duguet, "Pairing in finite nuclei from lowmomentum two- and three-nucleon interactions," in Fifty Years of Nuclear BCS (2013) pp. 229-242.

54. T. Duguet, T. Lesinski, K. Hebeler, and A. Schwenk, Mod. Phys. Lett. A 25, 1989 (2010).

55. T. Lesinski, K. Hebeler, T. Duguet, and A. Schwenk, J. Phys. G 39, 015108 (2012).

56. F. Barranco, R. Broglia, G. Colo, E. Vigezzi, and P. Bortignon, Eur. Phys. J. A 21, 57 (2004).

57. G. Gori, F. Ramponi, F. Barranco, P. F. Bortignon, R. A. Broglia, G. Colò, and E. Vigezzi, Phys. Rev. C 72, 011302 (2005).

58. A. Pastore, F. Barranco, R. A. Broglia, and E. Vigezzi, Phys. Rev. C 78, 024315 (2008).

59. A. Idini, F. Barranco, E. Vigezzi, and R. Broglia, J. Phys. Conf. Ser. 312, 092032 (2011).

60. T. Duguet, P. Bonche, P.-H. Heenen, and J. Meyer, Phys. Rev. C 65, 014310 (2001).

61. M. Bender, G. F. Bertsch, and P.-H. Heenen, Phys. Rev. C 73, 034322 (2006).

62. K. Van Houcke, F. Werner, E. Kozik, N. Prokof'ev, B. Svistunov, M. J. H. Ku, A. T. Sommer, L. W. Cheuk, A. Schirotzek, and M. W. Zwierlein, Nature Physics 8, 366 (2012).

63. R. F. Garcia Ruiz, M. L. Bissell, K. Blaum, A. Ekström, N. Frömmgen, G. Hagen, M. Hammen, K. Hebeler, J. D. Holt, G. R. Jansen, M. Kowalska, K. Kreim, W. Nazarewicz, R. Neugart, G. Neyens, W. Nörtershäuser, T. Papenbrock, J. Papuga, A. Schwenk, J. Simonis, K. A. Wendt, and D. T. Yordanov, Nature Physics 12, 594 (2016).

64. J. Simonis, S. R. Stroberg, K. Hebeler, J. D. Holt, and A. Schwenk, Phys. Rev. C 96, 014303 (2017).
65. T. Hüther, K. Vobig, K. Hebeler, R. Machleidt, and R. Roth, (2019), arXiv:1911.04955 [nucl-th] .

66. J. L. Friar, J. Martorell, and D. W. L. Sprung, Phys. Rev. A 56, 4579 (1997).

67. I. Angeli and K. Marinova, Atomic Data and Nuclear Data Tables 99, 69 (2013).

68. P. J. Mohr, B. N. Taylor, and D. B. Newell, Rev. Mod. Phys. 80, 633 (2008).

69. H.-W. Hammer and U.-G. Meißner, Science Bulletin 65, 257 (2020).

70. "CODATA recommended values of the fundamental physical constants: 2018," https://physics.nist. gov/cgi-bin/cuu/Value?rp.

71. A. J. Miller, K. Minamisono, A. Klose, D. Garand, C. Kujawa, J. D. Lantis, Y. Liu, B. Maaß, P. F. Mantica, W. Nazarewicz, W. Nörtershäuser, S. V. Pineda, P. G. Reinhard, D. M. Rossi, F. Sommer, C. Sumithrarachchi, A. Teigelhöfer, and J. Watkins, Nature Physics 15, 432 (2019).

72. W. Xiong et al., Nature 575, 147 (2019).

73. T. Miyagi, T. Abe, M. Kohno, P. Navrátil, R. Okamoto, T. Otsuka, N. Shimizu, and S. R. Stroberg, Phys. Rev. C 100, 034310 (2019).

74. R. Garcia Ruiz and A. Vernon, Eur. Phys. J. A 56, 136 (2020).

75. F. Barranco and R. Broglia, Physics Letters B 151, 90 (1985).

76. E. Caurier, K. Langanke, G. Martnez-Pinedo, F. Nowacki, and P. Vogel, Physics Letters B 522 , 240 (2001).

77. C. Barbieri, D. Van Neck, and W. H. Dickhoff, Phys. Rev. A 76, 052503 (2007).

78. T. Duguet, V. Somà, S. Lecluse, C. Barbieri, and P. Navrátil, Phys. Rev. C 95, 034319 (2017).

79. N. Rocco and C. Barbieri, Phys. Rev. C 98, 025501 (2018).

80. H. De Vries, C. W. De Jager, and C. De Vries, Atom. Data Nucl. Data Tabl. 36, 495 (1987).

81. P.-G. Reinhard and W. Nazarewicz, Phys. Rev. C 95, 064328 (2017).

82. A. Idini, C. Barbieri, and P. Navrátil, Phys. Rev. Lett. 123, 092501 (2019). 